\title{
Applications on Mobile Devices for Solving Derivative Problems
}

\author{
Elena F. Ruiz ${ }^{1}$, Laura I. Garay², Juan J. Gutiérrez ${ }^{3}$ \\ ${ }^{1}$ Instituto Politécnico Nacional, Dpto. de Posgrado, SEPI-ESCOM, Cd. de México, \\ Mexico \\ ${ }^{2}$ Instituto Politécnico Nacional, UPIITA, Cd. de México, \\ Mexico \\ ${ }^{3}$ Instituto Politécnico Nacional, ESCOM, Cd. de México, \\ Mexico \\ efruiz@ipn.mx, igaray@ipn.mx, jgutierrezg@ipn.mx
}

\begin{abstract}
This paper presents a set of 3 mobile applications where derivative concept is analyzed through everyday life examples of rate of change and optimization. Augmented reality and GeoGebra were used as computational tools. In the applications test, a sample of 30 students formed the control group (GC) and 30 student formed the study group (GE). Both have been applied the same initial and final questionnaire. The GE used the applications on their mobile devices, while the GC worked the same subject without them during 1 hour and a half in 5 sessions. Results for both groups in initial and final evaluation mean results on 2.08 to 3.30 and 2.58 to 7.47 for GC and GE respectively. Applying F test, a statistically significant difference in increase was found $(p<0.002)$. This results showed that GE identified the concept of derivative and correctly applied that concept to real problems solving correctly $74 \%$ of the final questionnaire, so it is concluded that these activities supported mobile devices open a new way in educational research.
\end{abstract}

Keywords: Classroom learning, mobile computing, educational research, augmented reality.

\section{$1 \quad$ Introduction}

Currently there are software programs that are directed to help understanding of mathematics, however, it was found that most of them help to develop algebraic and memorizing skills but visualization, understanding and reasoning mathematics are lacking [1]. These programs are generally designed to be used on a desktop computer by limiting the scope and availability of the software. Although there is a tendency to include this software in classrooms to integrate their capabilities to the tools for 
teachers in Mexico, it has been limited its introduction because classrooms don't have infrastructure and associated maintenance is required.

On the other hand, it is known that the population between 15 and 35 years old have a mobile phone and the trend of acquiring mobile devices is increasing [2,3] as well as their processing capabilities, visualization and internet availability so this situation makes mobile a viable tool to be include in the education process named m-learning [4].

In this paper, three mobile applications that are intended to help in different cognitive skills development during its use in classroom teaching. These applications were based on problems taken from textbooks [5, 6, 7], with some changes and programmed as interactive environments in a mobile software. The topic was the derivative and its use in situations where a rate of change and optimization is required $[8,9]$.

The first application, "Android balloon" used augmented reality concept, allowing to analyze a real situation, take measurements and use them to solve problems associated with what happens to an object when it undergoes a change in any of the variables that define (volume and radius of the sphere). The "building a box" and "clockwise" application used GeoGebra, a dynamic program that offers various representations of objects from each of its possible perspectives: graphical views, algebraic topics, and information organization in charts and spreadsheets, together with data sheets dynamically linked [10]. The application, called "trickle", uses the GPS positioning system with other sensors that owns the device to catch elements that could be analyzed by the student.

\section{$2 \quad$ Methodology}

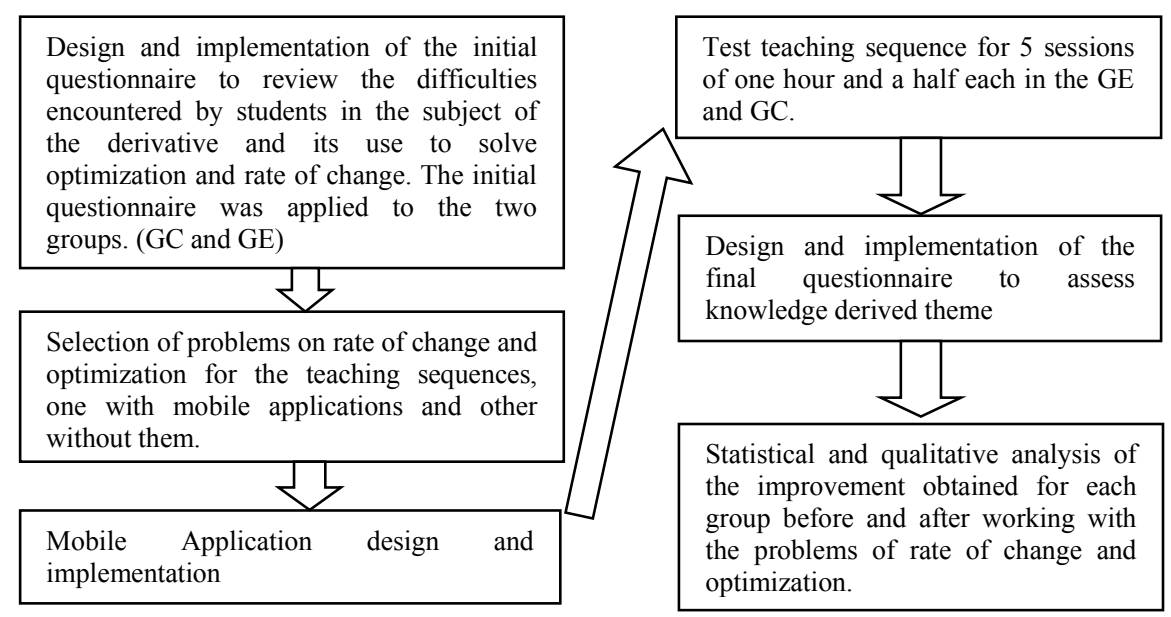

Fig. 1. Steps followed in the study. 
The application was tested in two groups, one called control group (GC) and the other study group (GE). Each group consisted of 30 students who were taking their first semester of studies in one of the units of the Instituto Politécnico Nacional. In order to evaluate if the mobile applications improved the learning of students after being working on the derivate topic and solving problems of rate of change and optimization, it was applied two questionnaires: one before the topic was studied named initial questionnaire, and a final questionnaire after finished the topic learning then it was compared the mean results obtained in both questionnaires. Two sequences were designed, one to be worked with the control group (GC) and the other for the study group (GE).

The teaching sequence worked with GC, was integrated with problems taken from textbooks for engineers [5], [6], [7], and were worked traditionally in the classroom. That is, the teacher wrote down information on the board, explained, solved it and the students copied in his notebook what has been done by the teacher.

The GE teaching sequence consisted of the same problems, included the mobile applications and teacher allowed students to interact with them and respond to the questions asked. The steps followed in this work are shown in the diagram of Figure 1.

\subsection{Design and Implementation of the Initial Questionnaire}

The diagnostic or initial questionnaire has 8 questions related to the learning Unit named Calculus and specifically to the issues of the derivative and their uses as the rate of change and optimization. Three of the 8 questions had incised so the total number of question to answer was 12 .

In Figure 2, examples of conceptual and algorithmic problem are shown.

Let be $L$ a tangent line to $y=f(x)$ on point $(5,3)$ as is shown in the figure. Find $f^{\prime}(5)$.

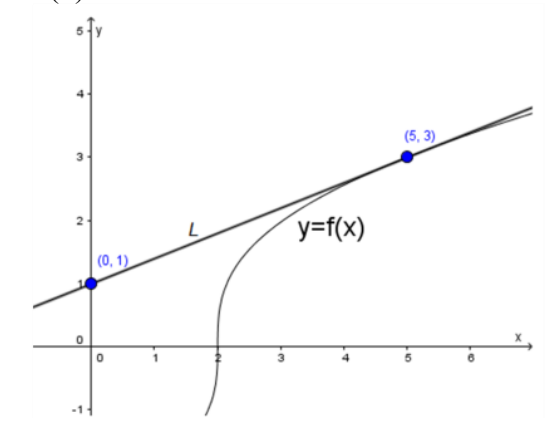

Calculate the derivative function at the indicated point. $y=x^{2}-4 x+1$ and $x=2$.

¿Which is the meaning of the result?

Fig. 2. Examples of the types of questions. On the left side: a conceptual problem and on the right side: an example of an algorithmic problem.

Questions were classified into two types: algorithmic and conceptual type. Algorithmic questions [8,9], are those in which the student must know the method or 
steps to solve the exercise and will only be asked to calculate or find a result. (Questions 2b, 3a, 5a, 5b and 5c). On the other hand, concept questions imply that the student has understood the concept involved $(1,2 \mathrm{a}, 3 \mathrm{~b}, 4,6,7, \mathrm{y} 8)$ and applies it to find the answer to the situation proposed in question.

\subsection{Selection of Problems}

Three problems were chosen from the textbook [6], [7], and then they were converted into interactive applications for students in the study group (GE). Those mobile applications are explained in order to show how they were generated. They were named: "Android balloon", "Building a box" and "Clockwise".

About the control group (GC) they worked the same problems, but without using the applications, instead of, the teacher explained using the blackboard and the students solved them using for this a calculator, and wrote the answers in their notebooks.

\subsubsection{Android Balloon}

This mobile application was intended to support the student understanding of the rate of change. "Android balloon" shows this phenomenon, using an example where a balloon with shape of android, it lost gas and how, as time goes by, the radio and surface changes.
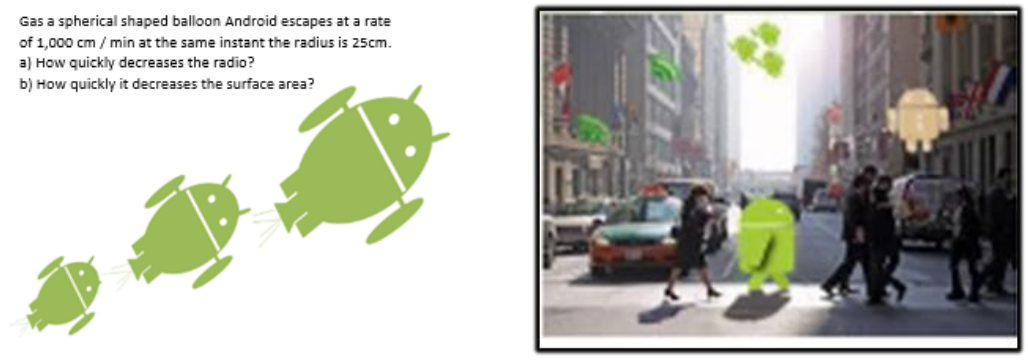

Fig. 3. Examples of the application where the object undergoes a change and can apply the concept of rate of change.

In this application, the student could see, in real time and in the classroom, what happens to an object when it undergoes to a change in an instant. This phenomenon is called rate of change and it can be quantified using the derivative concept. This activity used the camera of mobile device and a marker of augmented reality. The first one, for obtaining the scenario and the second one, to take the decision of where to display the globe. In order to keep the application as simple as possible and reduce the mobile requirements, the use of the location sensors of the device were avoided, even though it is common to use it in augmented reality applications. Instead of this, the markers or labels helped to place where the Globe should be and where, part of image was overlapped when an increase of Globe size have to be displayed. 
Although it is easy to create own marks, in this application we use the official one in order to allow students to reproduce this exercise by themselves. It was selected an easily identifiable image such as a black question mark on a white background so a quickly recognition of the marker allowed to determine the orientation and scale of the object to draw. The AndAR library was used with this purpose (Figure 3).

The student can stop the animation at different times, measure on the figure and then respond to questions with the obtained data.

\subsubsection{Construction of a Box with Maximum Volume}

The purpose of this application is to obtain the maximum volume of a box without a lid if a specific area was given. It was intended that the student visualizes the change in the volume of a box through different registers of representation of the volume function (algebraic, tabular and graphic register). In the algebraic register, the change in volume was observed by replacing the values of the independent variable " $x$ " in the analytical expression of the volume:

$$
V(x)=x *(\text { high }-x)(\text { width }-x) .
$$

In the tabular register, a volume change was observed when the $\mathrm{x}$ value in the table is changed then a change of the value of the variable $\mathrm{V}$ is observed. In the graphic register, if the curve changes then a modifying volume is presented.

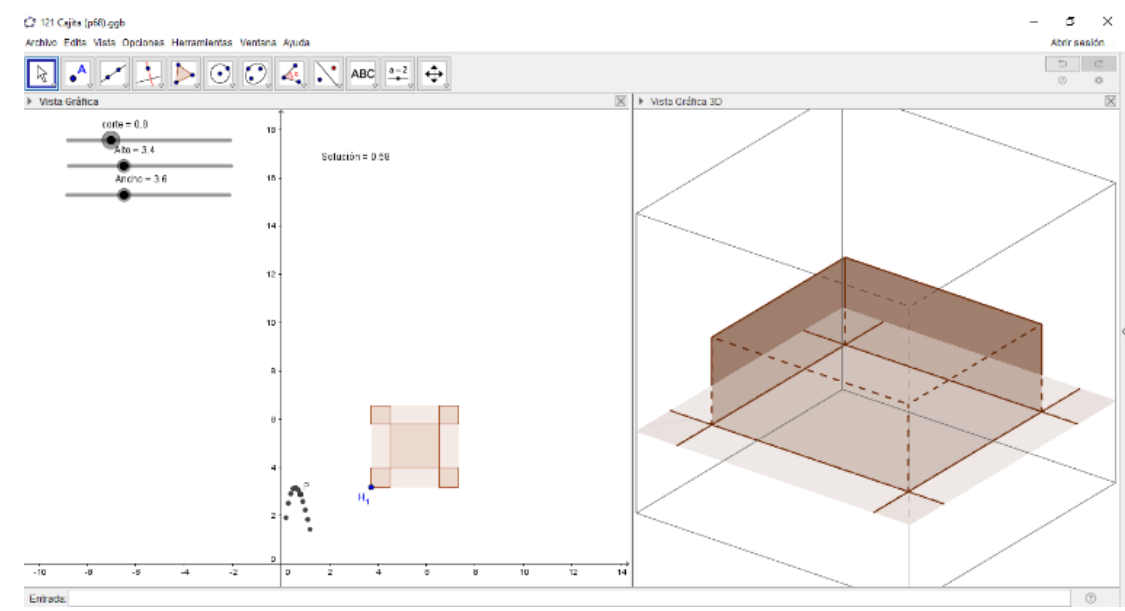

Fig. 4. Application for analyzing volume optimization of a box based on a sheet and a side cut $\mathrm{x}$. It contains: sliders on the left side, in the middle area, the volume (y-axis) with respect to the cut ( $\mathrm{x}$ axis) and the sheet in which is presented the cut to be made. Finally, a 3D view of the box in the right side.

For the construction of this mobile application, the free GeoGebra software was used [10]. As is shown in Figure 4, to build the simulation of this problem, slide bars controls were created for the height and width variable of the sheet that are shown in the center as a polygon. Four boxes are used to show the cuts that have to be 
performed. It has a point $\mathrm{H}_{-} 1$ from which both the whole picture and the boxes generated by the cut. In the first case the image is generated by adding the coordinates of this point to the width and length of the sheet, which also can be modified using the sliders on the left side and in the second case, represents the independent variable.

In the $3 \mathrm{D}$ graph, the sheet is located in the $\mathrm{z}=0$ plane and the cut sheet has been folded up to conform the complete box. When the sliders are moved, the changes are reflected in the other zones of the window. It can also be observed the point $\mathrm{P}$ whose coordinates are $\mathrm{x}$, the cut dimension and the volume of the box $\mathrm{V}(\mathrm{x})$ computed.

Student can change the scales, see the generated data in a spreadsheet and images so he can explore different representations that could help to understand the concepts and solve the problems.

\subsubsection{Clockwise}

In this application, the student is asked to find the speed of changes in the distance between the tips of the hands of a clock after a lapse of time and find some of the extreme values such as maximum and minimum.

He solves this problem using GeoGebra again, a response windows is shown in Figure 5. The hands of the clock were made with two line segments, one of greater length such as in a clock. One segment is entirely free, representing the minute hand and the other hand position is calculated as a fraction of the angle and the minute rate with the vertical position as reference.

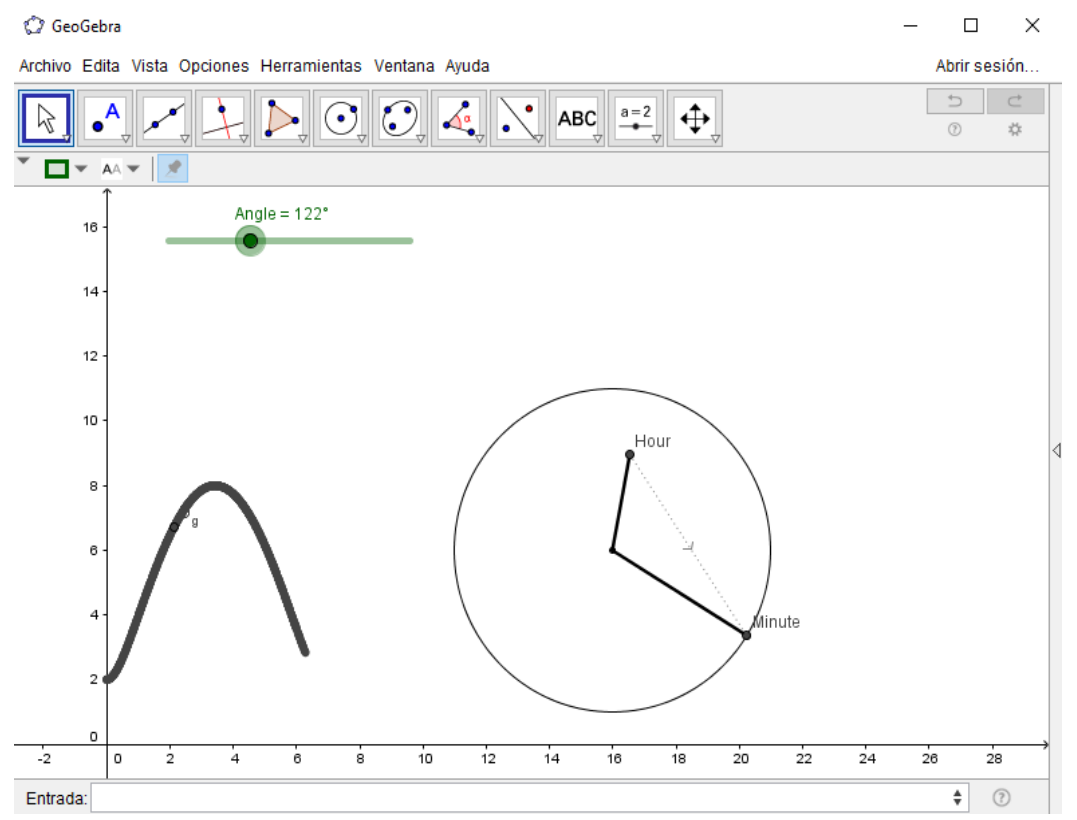

Fig. 5. Clockwise. The circle on the right represents the clock and the two line segments, the hands. The graph on the left shows how the distance between the handles changes the angle that makes the minute hand with the vertical. 
The obtained data is presented in animation and the average rate of change and approaching the instantaneous rate of change is requested. So the user could observe and find the need data just checking the animation and thinking about the meaning of the function graph and the derivative.

\section{Results}

The initial questionnaire was a diagnostic and was graded using right or wrong (in the case of not answering the question was taken as incorrect). The questions were focused on reviewing both conceptual and algorithmic abilities of the students and found that most of them showed gaps related to the derivative concept.

There were 12 questions in total, 5 evaluated the algorithmic problems and 6 the conceptual problems. The graph in Figure 6 shows the percentage of correct answers for each question, the questions do not appear in the order in which students answered, but have been divided into conceptual and algorithmic type.

It can be seen that both the control group (GC) and the study group (GE) have the same performance in the initial questionnaire, which is low. It was demonstrated by grouping together the results of the 60 students of the initial questionnaire and calculating the student's t-test, along with the Mann-Whitney test showing that they are not different with $\mathrm{p}<0.05$. GC averaged was 2.02 in the initial questionnaire and GE obtained an average of 2.58 .

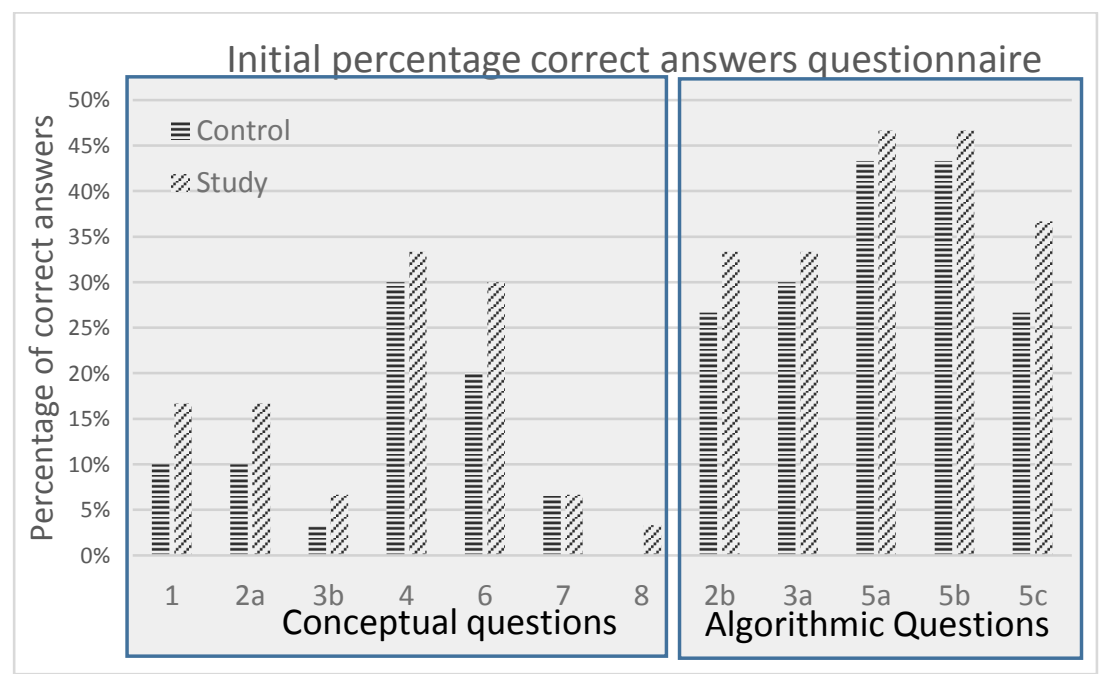

Fig. 6. Results of both groups, showed how algorithmic questions are answered correctly more often than the others in initial questionnaire.

Considering only the average of algorithmic questions and comparing to the average of conceptual questions is observed that in the case of algorithmic questions, the control group obtained 3.40 and 3.93 mean grade. But in conceptual questions 
averages was 1.14 for the GC and 1.96 for GE. So both groups showed deficiencies, especially about the application of the concept of derivative.

The more frequently error detected is that students replaced the given values in the mathematics formula without noticed what the exercise was asked to respond. The second most common mistake was to confuse the concepts they were asked.

After working with the traditionally teaching sequences in GC and in the GE using the mobile applications, a final questionnaire was conducted in order to evaluate if there is a different performance between students of GC and GE.

Both groups improve their performance. In the GC, the average grade increment was from 2.08, which was obtained in the questionnaire diagnosis, to 3.30 in the final questionnaire. In the case of GE, the average number of correct answers changed from 2.58, which was obtained in the diagnostic questionnaire to 7.47 that was obtained in the final questionnaire. When comparing the two groups with the $\mathrm{F}$ test, it was observed that the increases are different with $\mathrm{p}<0.002$.

On the other hand, the results of analyzing each of the questions grouped by the concept and algorithmic type are shown in Figure 7.

In both groups, the percentage of correct answers is higher for the questions algorithmic regarding conceptual type, indicating that students are better following a steps set or memorizing the derivation rules than in the construction of the concept of the derivative.

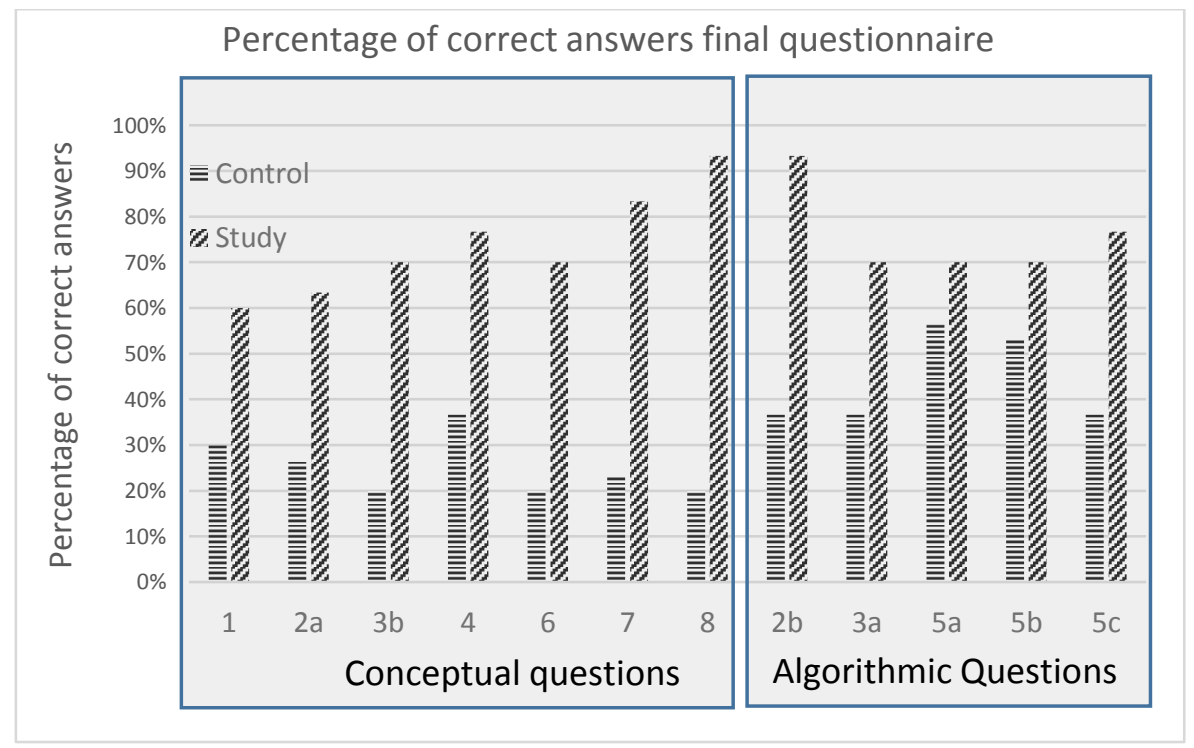

Fig. 7. Questions by type answered correctly in control and study group in final questionnaire.

Whereas in the control group, the teacher drew static graphs on the board, in the study group, the student used dynamic graphics through the mobile applications and it was noted that the interactions with cellphone mainly, zooms, interaction with the mathematical model and visualization of the effects, positively contributed to student performance. 


\section{Conclusions and Future Work}

It was observed that the students of the control group still required to know the algebraic expression of the function derivative in order to substitute the data on it. On the other hand, students in the study group were able to recognize that the function derivative value is the slope of the tangent at the given point, and thus remembered the definition of a line by two points so they could answer correctly.

It was observed that most of the student had cellphones, then these applications are feasible and could be integrate in the education system because this concept strengthen the learning skills promoted in the IPN. In reviewing applications and activities developed in this research, it was observed that with a mobile device with current performance, ubiquity is achieved and m-learning could be used in education.

Acknowledgments. This work was supported by Instituto Politécnico Nacional (IPN, México) with grant SIP20150296. The authors wish to thank COFAA for the incentive compensation provided.

\section{References}

1. Johnson, L., Adams, S., Cummins, M., Estrada, V., Freeman, A., Hall, C.:. NMC Informe Horizon 2016. Edición Educación Superior de Austin, Texas: The New Media Consortium.donado, pp. 36 (2016)

2. Pew Research Center.: The Smartphone Difference. Available: http://www.pewinternet. org/2015/04/01/us-smartphone-use-in-2015 (2015)

3. Kurkovsky, S.: Integrating mobile culture into computing education. In: Integrated STEM Education Conference (IEEE), No. 9, pp. 1,4 (2012)

4. Josh, M., Somsak, S.: Developing Mobile Learning Applications for Electrical Engineering Courses. Department of Engineering TN, U.S.A., University of Tennessee Martin, Tennessee (2011)

5. Sokolowski, A., Yalvac, B., Loving, C.: Science modeling in pre-calculus: how to make mathematics problems contextually meaningful. International Journal of Mathematical Education in Science and Technology (2011)

6. Stewart, J.: Cálculo de una variable. Trascendentes tempranas, Sexta Edición (2011)

7. Larson, R. E., Hostetler, R. P., Edwards, B. H.: Cálculo y Geometría Analítica. Sexta Edición, Editorial McGraw Hill (2014)

8. Aravena, M.: Las principales dificultades en el trabajo algebraico. Un estudio con alumnos de ingeniería de la U.C.M. Revista Académica Universidad Católica del Maule, No. 28, pp. 63-81 (2002)

9. Hit, F., Dufour, S.: Un análisis sobre la enseñanza del concepto de la derivada. En: C. e. Cuevas, La enseñanza del cálculo diferencial e integral, México, Pearson, pp. 19 (2013)

10. Caligaris, M., Schivo, M., Romiti, M.: Calculus \& GeoGebra, an Interesting Partnership. Procedia - Social and Behavioral Sciences, Available at: http://dx.doi.org/10.1016/ j.sbspro.2015.01.735, Vol. 174, pp. 1183-1188 (2015) 